\title{
AN INTRODUCTORY REVIEW
}

\author{
M.J. SEATON \\ (University College London, England)
}

\begin{abstract}
The actors are at hand; and, by their show, You shall know all that you are like to know. (Shakespeare, A Midsummer Night's Dream)
\end{abstract}

The Organising Committee of this Symposium agreed that we should begin with a prologue and end with an epilogue. The first task of a prologue is to introduce the actors: we have The Eskimo, The Owl, The Helix and The Dumb-Bell (Figure 1) and many more. The dramatis personae of all known galactic planetary nebulae has been prepared for us by Dr Perek and Dr Kohoutek, and we are all much indebted to them for their labours. Planetaries in the Magellanic Clouds will be introduced by $\mathrm{Dr}$ Westerlund.

Planetary nebulae are easily recognised, they can be observed to great distances and they belong to an interesting intermediate population type. The galactic distribution of planetaries, which will be discussed by Perek, is of great importance for studies of galactic structure; thus of all objects which can be observed in the visible spectrum, the planetaries provide the best means of determining the direction to the galactic centre. The problem of determining distances is one of great importance and of considerable difficulty. It is closely related to the problem of determining the masses of the nebulae.

Consider a uniform spherically symmetric nebula of radius $R$, ionized mass $M_{\mathrm{i}}$ and surface brightness $S$ in a hydrogen line. It may be shown that

$$
R=K S^{-1 / 5} \text {, }
$$

where $K$ is proportional to $M_{\mathrm{i}}^{2 / 5}$. The distance is $r=R / \theta$, where $\theta$ is the angular radius. Aller and Minkowski first pointed out that the distance could be calculated if a value of $M_{\mathrm{i}}$ is assumed. If a nebula is optically thin for ionizing radiation the ionized mass $M_{\mathrm{i}}$ is equal to the total nebular mass $M$. Shklovsky showed that it was plausible to assume that planetaries will become optically thin at some later stage in their evolution, and further assumed that the process of mass ejection is complete at this stage, so that $M$ remains constant, and that the same value of $M$ can be used for all nebulae. Using these ideas, he was able to draw some very interesting conclusions concerning the evolution of the nebulae and of their central stars. Further studies 

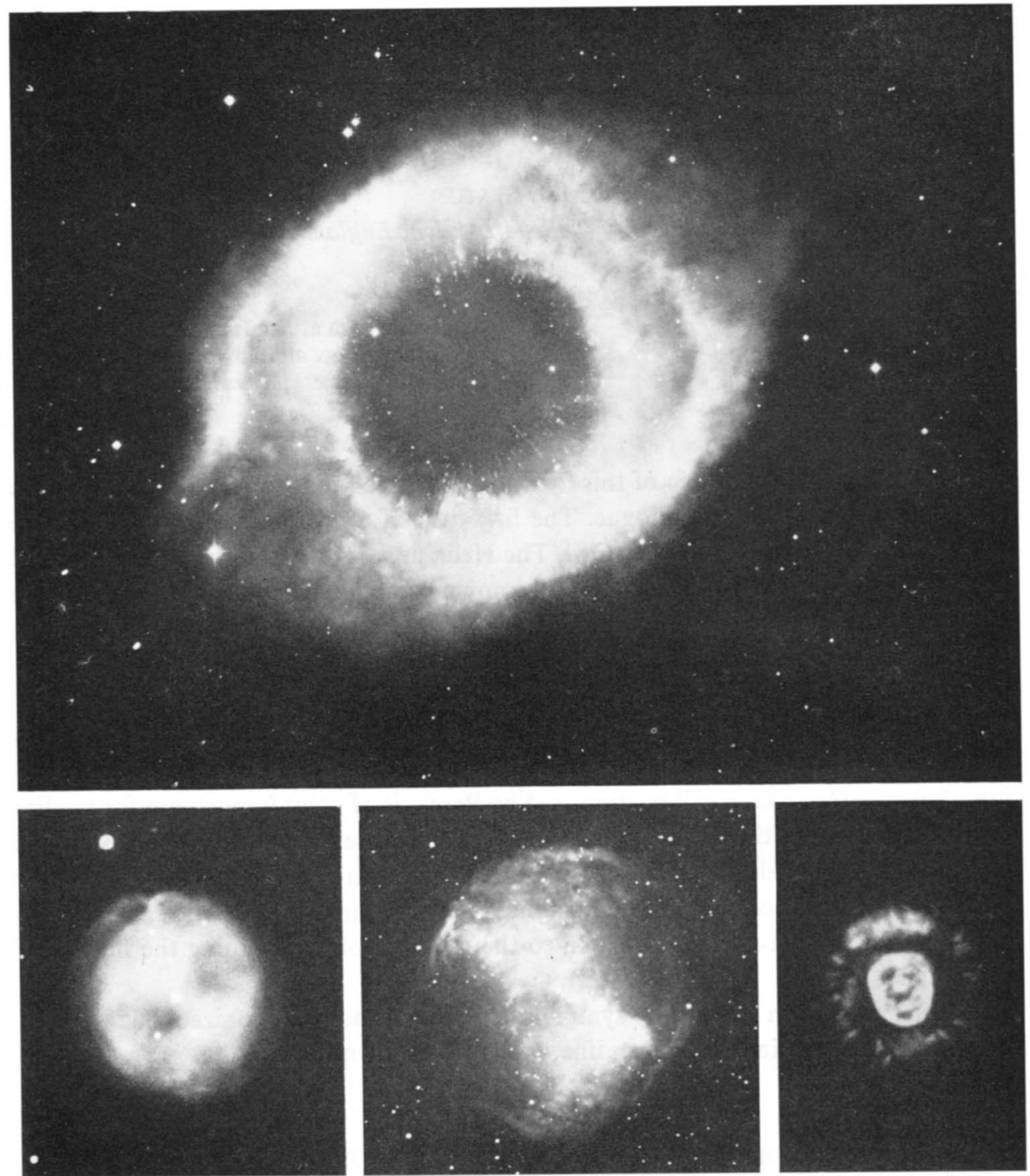

Fig. 1. Top, NGC 7293, the Helix Nebula. Lower left, NGC 3587, the Owl Nebula. Lower middle, NGC 6853, the Dumb-Bell Nebula. Lower right, NGC 2392, the Eskimo Nebula. All Mount Wilson and Palomar Observatory photographs.

were made by O'Dell, who calculated $N(R)$, the number of planetaries per unit volume per unit radius interval. This quantity is of importance for discussions of the origin and final evolution of the nebulae. For optically thin nebulae the expansion velocity $\dot{R}$ should be equal to the expansion velocity $V$ measured using the Doppler effect. 
Since $V$ is practically constant it is to be expected that $N(R)$ should be a constant, independent of $R$, for optically thin nebulae. Using Šklovsky's method, O'Dell found $N(R)$ to be constant within a certain range of values of $R$, and subsequent work by Harman and Seaton showed that this was precisely the range for which the nebulae could be expected to be optically thin. It therefore appears that Sklovsky's method can be relied upon for the determination of the distances of optically thin nebulae. The big question which remains is that of accurately calibrating the distance scale, that is, of determining the constant $K$. Different estimates of $K$ differ by factors of 2 or more, and the absolute value of $N(R)$, which is proportional to $K^{-4}$, is therefore very uncertain. I hope that this question of calibrating the distance scale will be discussed in some detail during the Symposium.

Our second Session is concerned with observations of the spectra of planetaries. The very first paper on this subject was written by Huggins in 1864. Having completed extensive studies of laboratory, solar and stellar spectra, Huggins explains why he turned his attention to the nebulae:

"It became therefore an object of great importance, in reference to our knowledge of the visible universe, to ascertain whether this similarity of plan observable among the stars, and uniting them with our sun into one great group, extended to the distinct and remarkable class of bodies known as nebulae. ... Some of the most enigmatical of these wondrous objects are those which present in the telescope small round or slightly oval discs. For this reason they were placed by Sir William Herschel in a class by themselves under the name of Planetary Nebulae."

The stellar spectra observed by Huggins were characterized by continua and by dark absorption lines. He describes his first observation of a planetary nebula, NGC 6543:

"On August 29th, 1864, I directed the telescope armed with the spectrum apparatus to this nebula. At first I suspected some derangement of the instrument had taken place; for no spectrum was seen, but only a short line of light perpendicular to the direction of dispersion. I then found that the light of this nebula, unlike any other extra-terrestrial light which had been subjected by me to prismatic analysis, was not composed of light of different refrangibilities, and therefore could not form a spectrum. A great part of the light from this nebula is monochromatic."

On using a smaller slit width, Huggins was able to observe a number of lines and to identify the $\mathrm{H} \beta$ line of hydrogen. He was not able to identify the stronger lines but he speculated that they might "indicate a physical difference in the atoms".

It was not until 1928 that the nebular lines were identified by Bowen as due to forbidden transitions in ions of oxygen, nitrogen and other common elements. I have always felt that Bowen's achievement was remarkable in that, in addition to making the identifications, he gave a very clear discussion of the basic physical processes taking place. At about the time that this work was done, pioneering studies of physical processes in nebulae were begun by Zanstra and by Menzel. With great physical 
insight they were able to develop methods for the determination of star temperatures, at a time when a quantitative theory of the hydrogen recombination spectrum had not yet been worked out. Observational data required for the application of these methods were first obtained by Zanstra. A series of papers by Menzel and his collaborators, on Physical Processes in Gaseous Nebulae, has made a contribution to our subject of fundamental importance.

A great deal of effort is still being devoted to work on nebular photometry, and I would particularly mention the very extensive observations made by VorontsovVelyaminov and his collaborators at the Sternberg Astronomical Institute, and the accurate spectrophometry of individual objects made by Aller and his collaborators. Flux measurements for a considerable number of planetaries have now been made at radio wavelengths, and the conclusions which can be drawn from them will be discussed during the Symposium. Within the near future we may also expect to have observations in the far infrared and in the ultraviolet, and two of our participants have had the courage to accept invitations to make predictions about the results which may be obtained.

Interest in planetary nebulae has led to many pioneering investigations in atomic physics, plasma physics and radiative transfer theory, and the results obtained have been important for many other branches of astrophysics and laboratory physics. In discussing these questions of basic physical processes, in the third session, we should remember that our main concern at this Symposium should be in the astrophysical significance of the results which have been obtained. Better observations and more detailed quantitative studies of physical processes enable us to determine much better chemical composition of the nebulae. We must consider not only the reliability of the results, but also their significance; one may ask, for example, whether the material ejected to form a planetary is contaminated by material which has undergone nuclear reactions during the evolution of the central star.

Our 4th Session will be concerned with questions relating to the structures and dynamics of the nebulae. These may provide important clues to understanding the processes which lead to mass ejection and formation of planetaries. We may also expect that there will be a great deal of interest in the possible importance of magnetic fields. New observations have been made, using interferometric and high-dispersion techniques, which provide a great deal of new information on velocity fields in nebulae.

What can be deduced from the spectra of the central stars? Why do some of these stars have emission lines? To what extent can one justify the conventional assumptions made in model atmosphere calculations? How reliable are attempts to determine temperatures and luminosities of the central stars, and to deduce from observations the evolutionary tracks on the Hertzsprung-Russell diagram? These are some of the questions with which we shall be concerned in the 5th Session.

It has long been recognised that the nebulae evolve in a time of order $10^{4}$ years. This time is obtained on dividing a typical nebular radius by a typical expansion 
velocity. The work of Šklovsky and of O'Dell has shown that the central stars also undergo considerable evolution in times of this order, and it has recently been recognised that it is possible to observe directly the variabilities in individual nebulae and central stars, within time intervals of order 10 years. Questions of the origin and evolution of the nebulae and of their central stars will be discussed in Session 6. By now it seems to be well established that planetaries evolve towards the white dwarfs, but the nature and the time-scale of this evolution must be discussed, and we must consider whether all white dwarfs are formed from planetaries, or whether planetaries produce only some special group of white dwarfs.

One of the major outstanding problems which we have to consider is that of the origin of planetary nebulae. Many different topics discussed during the Symposium may give important clues to the solution of this problem.

Our epilogue will be spoken by a chorus, led by Dr Osterbrock. Doubtless there will be some discordant notes, but I hope that there will be some harmony as well. At the very end, we may have to quote once again from Shakespeare, from the epilogue to King Henry the Eighth:

'Tis ten to one this play can never please

All that are here: some come to take their ease,

And sleep an act or two; but those, we fear,

W'have frighted with our trumpets...

In calling for our play to begin, may I ask that all our speakers should let their trumpets sound loud and clear. 